\title{
Sistema de Hardware/Software para Estimativas de Perdas de Geração em Usinas Solares Fotovoltaicas
}

\author{
Guilherme G. Souza ${ }^{1}$, Ricardo R. Santos ${ }^{1}$, Erlandson F. Saraiva ${ }^{2}$ \\ ${ }^{1}$ Faculdade de Computação (FACOM) \\ ${ }^{2}$ Instituto de Matemática (INMA) \\ Universidade Federal de Mato Grosso do Sul - UFMS \\ Av. Costa e Silva, s/n $\mathbf{n}^{\circ}$ 79.070-900 - Campo Grande/MS - Brasil \\ \{guilherme.gloriano, ricardo.santos, erlandson.saraiva\}@ufms.br
}

\begin{abstract}
Soiling on the photovoltaic modules has a straight impact on the solar energy generation. One way to minimize such impact is to identify the power generation losses due to soiling and perform cleanup procedures. Towards this end, this work presents a hardware/software system comprised of an electronic platform for the acquisition of environmental variables and a non-linear mixed effect model (NLME) to estimate the power generation from the solar power plants. The system allows users to analyze the soiling impact on energy generation on photovoltaic solar plants. The model was evaluated using a real dataset comprised of measurements of generated power and environmental variables from October 2019 up to April 2020. The fitted model has presented a mean square error of 0.0044 and the results showed daily power losses below $1.1 \%$.
\end{abstract}

Resumo. A sujidade nos módulos fotovoltaicos tem um impacto direto na geração de energia solar. Uma forma de minimizar esse impacto é identificar as perdas na geração de energia devido à sujidade e acionar procedimentos de limpeza. Este trabalho apresenta um sistema de hardware/software composto por uma plataforma eletrônica para aquisição de variáveis ambientais e um modelo não-linear de efeitos mistos (NLME) para estimar a geração de energia das usinas solares. Esse sistema possibilita que usuários possam analisar as perdas de geração em função da sujidade em usinas solares fotovoltaicas. $O$ modelo foi avaliado usando um conjunto de dados real composto de medições de energia gerada e variáveis ambientais de Outubro/2019 a Abril/2020. O modelo ajustado apresentou um erro quadrático médio de 0,0044 e os resultados mostraram perdas diárias de energia abaixo de 1, $1 \%$.

\section{Introdução}

Os sistemas Fotovoltaicos (FV) estão entre as fontes de geração de energia elétrica mais promissoras, pois sua tecnologia está em constante evolução, melhorando a eficiência e produtividade, reduzindo custos e mantendo um baixo impacto ambiental [Catelani et al. 2012]. O Brasil possui um grande potencial para utilização de usinas $\mathrm{FV}$, apresentando uma boa incidência solar e pouca variabilidade anual 1 . Entretanto, um

\footnotetext{
${ }^{1}$ Relatório do Ministério de Minas e Energia do Brasil
} 
desafio complexo que permanece para a indústria dessa área é a manutenção do desempenho na presença de condições ambientais adversas.

Em sistemas FV, um elemento que afeta as perdas de geração de energia é a sujidade - acúmulo de partículas de poeira e outros materiais suspensos na atmosfera - sobre os módulos fotovoltaicos. Considerando as variáveis ambientais e seus impactos na geração de energia solar, a sujidade é, depois da irradiância solar e da temperatura ambiental, o terceiro motivo de perdas de energia em usinas de energia solar [Gostein et al. 2014].

Estudos sobre perdas de geração de energia devido à sujidade revelam perdas mensais de $14 \%$ até $20 \%$ [Zorrilla-Casanova et al. 2011]. Entender o padrão de deposição da sujidade é crucial para fornecer soluções para este problema. Existem várias propostas para caracterizar as perdas de sujidade [El-Shobokshy and Hussein 1993], em diferentes tecnologias de módulos [Mani and Pillai 2010]. Mesmo com diversos trabalhos existentes, existe uma lacuna no que diz respeito a técnicas e metodologias para detecção de sujidade e avaliação de seu impacto na geração de energia em plantas fotovoltaicas.

Este trabalho apresenta o desenvolvimento de uma solução de hardware e software para a identificação da sujidade e estimação de seu impacto na geração de energia solar fotovoltaica. A solução envolve o projeto e desenvolvimento de uma estação eletrônica para aquisição de dados ambientais e um preditor baseado em um modelo não-linear log-logístico de efeitos mistos, para estimar a geração energética e possibilitar a análise de perdas devido à deposição de sujidade. O uso conjunto de preditor que utiliza modelo não-linear log-logístico e uma infraestrutura de coleta e envio de dados ambientais e de produção ainda não foi observado na literatura da área. Ressalta-se também que esta pesquisa estende um trabalho anterior desenvolvido pelo grupo de pesquisa [Ribeiro et al. 2021] que propôs um modelo um modelo de linear multivariado e metodologia para estimativa de perdas por sujidade com aplicação em Usinas Solares Fotovoltaicas em Irecê-Bahia.

\section{Trabalhos Relacionados}

Os autores em [Mehta et al. 2018] determinam o impacto da sujeira, em painéis solares, utilizando uma Rede Neural Convolucional (CNN) tomando como entradas imagens RGB de painéis solares e variáveis ambientais como entradas. A abordagem estima a localização e o tipo de sujeira. O conjunto de dados rotulado foi construído usando imagens de dois painéis solares idênticos dispostos lado a lado e a porcentagem de perda de energia em relação ao painel de referência.

Um estudo abrangente sobre o impacto da sujeira em plantas fotovoltaicas em regiões do Oriente Médio e Norte da África foi estudado em [Hammad et al. 2018]. Os autores realizaram uma revisão de vários estudos científicos e destacaram o uso de modelos matemáticos a partir técnicas de regressão e redes neurais para estimar o impacto da sujeira nas perdas de energia e o momento ideal para limpar os painéis solares. Os modelos consideraram o tempo de exposição à poeira e a temperatura ambiente como variáveis independentes e a eficiência de conversão do sistema fotovoltaico como variável dependente.

Os estudos mencionados, assim como vários outros existentes na área [Pavan et al. 2013, Javed et al. 2017], adotaram conjunto de dados rotulados 
com informações de sujeira para aplicar seus métodos. Em grandes usinas solares fotovoltaicas, onde não existem tais informações disponíveis para todos os módulos, painéis ou matrizes fotovoltaicas, esses procedimentos necessitam de considerável intervenção humana. Além disso, algumas abordagens dependem de procedimentos manuais frequentes que consistem na limpeza de um módulo solar de referência (limpo) e na comparação com outro módulo (sujo), inviabilizando assim a aplicação para uma tomada de decisão dinâmica e com precisão.

A proposta deste trabalho, por outro lado, é baseada na aquisição automática de dados ambientais e de produção de energia e na utilização desses dados em um modelo de preditor não-linear. Tal sistema de hardware e hardware não demanda intervenção humana uma vez que a partir da plataforma eletrônica que coleta e envia dados para um ambiente de nuvem computacional, o modelo realiza as estimativas de geração energética. Diante dessas estimativas, um usuário pode analisar diferenças entre a produção energética real e estimada e, como consequência, tomar decisões embasadas sobre manutenções (limpeza) sobre os módulos da usina solar FV.

\section{Infraestrutura de Hardware para Coleta de Particulados}

Para aquisição contínua de dados ambientais foi projetada e desenvolvida uma infraestrutura de hardware composta por sensores, microcontroladores e sistemas de comunicação sem fio, denominada de estação de sujidade. Essa estação tem como função o envio de dados coletados para um sistema de monitoramento e supervisão (englobando também a predição de geração energética) em nuvem computacional 2

A estação de sujidade projetada utiliza um microcontrolador ESP32 (Espressif Systems), para executar sua rotina de aquisição e posteriormente transmissão de dados para um dispositivo gateway de comunicação chamado de Unidade Terminal Remoto (RTU - Remote Terminal Unit). A Figura 1 representa o projeto da plataforma eletrônica responsável pelo controle e operação da estação. A Figura 1a representa o circuito eletrônico (em uma placa de circuito impresso) que condiciona os componentes da estação de sujidade. A Figura $1 \mathrm{~b}$ representa os componentes eletrônicos principais responsáveis pelo interfaceamento e controle dos sensores conectados na plataforma eletrônica.

$\mathrm{Na}$ Figura 1b também é possível observar o conjunto de elementos agrupados em blocos. A organização de cada bloco foi realizada da seguinte maneira:

- uControler: Neste bloco está alocado o microcontrolador ESP32. Nele é possível verificar quais pinos estão sendo utilizados para conectar os sensores e demais componentes. Este microcontrolador foi alimentado com $5 \mathrm{~V}$ de tensão (pino VIN).

- Voltage Regulator 5V: Como a alimentação da placa eletrônica é realizada por uma fonte de $12 \mathrm{~V}$, o componente deste bloco faz a regulagem e controle da tensão que será fornecida ao microcontrolador ESP32. Este bloco utiliza como componentes: um regulador de tensão LM7805, para fixar a tensão de saída em $5 \mathrm{~V}$ e dois capacitores $(0,1 \mu \mathrm{F}$ e $0,33 \mu \mathrm{F})$ para seu funcionamento.

- Voltage Regulator 3V3: Este bloco faz a regulagem da tensão para 3,3V, que foi utilizada como tensão de entrada da maioria dos sensores e do relógio de tempo

\footnotetext{
${ }^{2}$ Disponível em http://solar2.facom.ufms.br/
} 


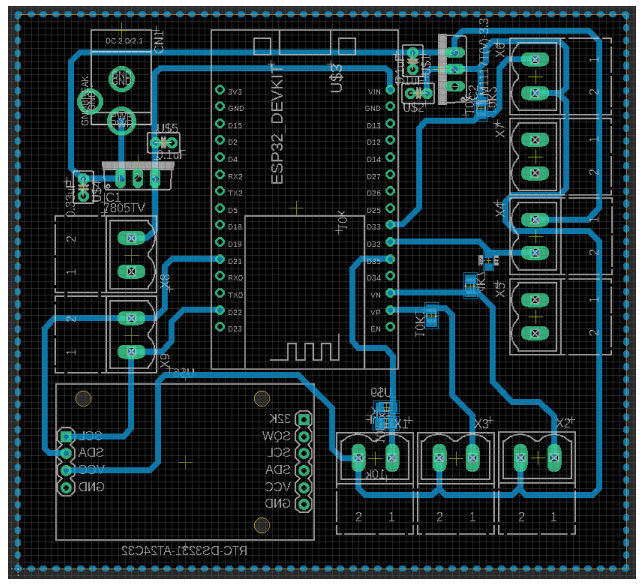

(a) Projeto da placa de circuito impresso.

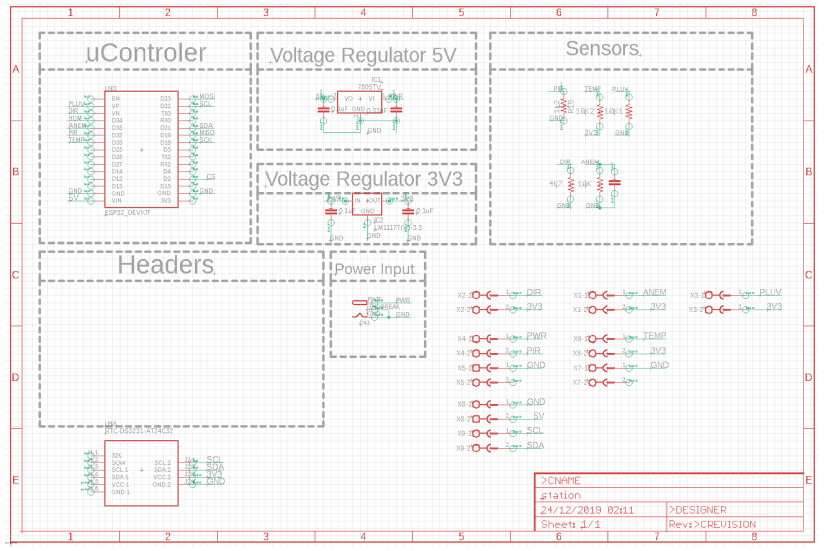

(b) Esquemático dos componentes eletrônicos da placa confeccionada.

Figura 1. Projeto do circuito eletrônico de controle da estação de sujidade.

real. Neste bloco foram utilizados um regulador de tensão LM1117 e dois capacitores (ambos de $0,1 \mu \mathrm{F}$ ), garantindo uma saída fixa de 3,3V.

- Sensors: Bloco utilizado para agrupar os circuitos condicionadores de sinais provenientes dos sensores, nele está presente os pinos dos sensores:

- PIR: O único sensor que necessita de tensão superior a 6V para o funcionamento é o piranômetro, sendo portanto, conectado diretamente na tensão fornecida pela fonte de alimentação (12V). Para condicionar este sensor foi utilizado apenas um resistor de pull-down ${ }^{3}$ de $1,2 \mathrm{k} \Omega$.

- TEMP: Para condicionar o sensor de temperatura foi utilizado um resistor de pull-up $\rfloor^{4}$ de $10,2 \mathrm{k} \Omega$. A tensão de alimentação deste sensor é de 3,3V, sendo fornecida pelo regulador de tensão.

- PLUV: No pluviômetro foi utilizado a alimentação de 3,3V e um resistor de pull-down de $10,1 \mathrm{k} \Omega$.

- DIR: No sensor de direção do vento, houve a necessidade apenas de um resistor de pull-down de $4,7 \mathrm{k} \Omega$. Este sensor também é alimentado pela tensão de 3,3V.

- ANEM: O anemômetro (sensor de velocidade do vento) é o único que além de utilizar um resistor, também faz uso de um capacitor para condicionar corretamente a sua saída. Neste sensor é utilizado um resistor de pull-down de $10 \mathrm{k} \Omega$ e um capacitor de $10 \mathrm{nF}$. Este circuito também é alimentado pelo regulador de tensão que fornece $3,3 \mathrm{~V}$.

Após o projeto e confecção da placa de circuito impresso para conexão dos sensores com os dispositivos eletrônicos, todos os componentes supramencionados foram conectados na mesma, resultando na estação de sujidade atual. A rotina de funcionamento da estação (Figura 2) apresenta atividades que foram implementadas em seu firmware.

\footnotetext{
${ }^{3}$ Resistores pull-down são usados para manter a entrada em nível lógico baixo quando nenhum outro componente estiver conduzindo.

${ }^{4}$ A ideia de um resistor pull-up é que ele fracamente "puxe" a tensão do condutor que ele está conectado para nível lógico alto. Contudo, o valor do resistor é intencionalmente alto o suficiente para que, se qualquer outra coisa que puxe fortemente a tensão do condutor para $0 \mathrm{~V}$, a tensão irá para $0 \mathrm{~V}$.
} 
A cada segundo, os dados ambientais são coletados via sensores, recebidos pelo subsistema de processamento e controle (plataforma microcontrolada) que processa essas informações, organiza os dados em um formato .json ${ }^{5}$ e encaminha para o dispostivo de terminal remoto responsável pela comunicação com o sistema em nuvem.

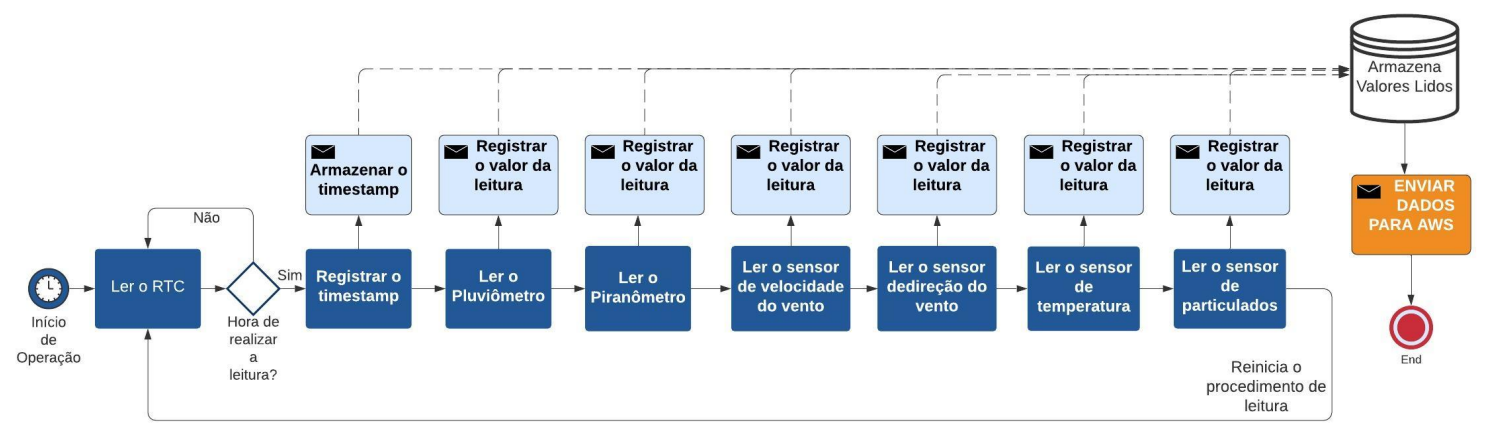

Figura 2. Diagrama BPMN $\sqrt{6}$ do fluxo de trabalho da estação de sujidade.

A estação de sujidade (Figura 3 ) foi instalada próxima a unidade fotovoltaica (UFV-UFMS) situada na Universidade Federal de Mato Grosso do Sul (UFMS) em Campo Grande-MS (Latitude: -20³0'39.09", Longitude: -54³7'11.394"). A UFVUFMS possui uma string de geração fotovoltaica composta por 38 módulos solares da tecnologia silício policristalino (p-Si), com potência nominal de $10,45 \mathrm{kWp}$. O inversor instalado é o modelo PRIMO 8.2-1 da Fronius International ${ }^{\odot}$.

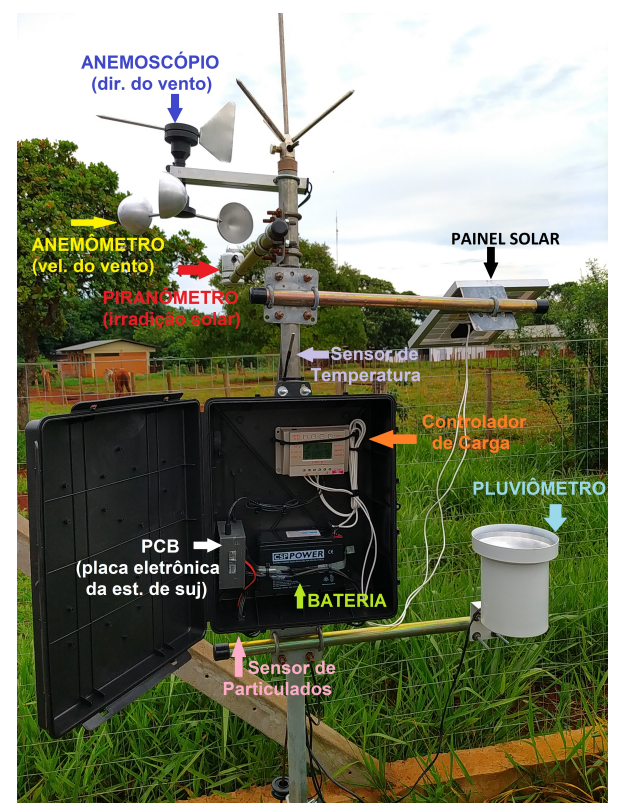

Figura 3. Estação de Sujidade: versão atual.

\section{Modelagem de Massa dos Particulados do Ambiente}

Uma forma direta para observar o efeito da sujidade sobre a geração FV é analisar a presença de particulados suspensos na atmosfera. Nesse sentido, deve-se, primeiramente, estimar a massa dessas partículas na superfície dos módulos FV. Com isso, alguns

\footnotetext{
${ }^{5}$ JSON - JavaScript Object Notation.
} 
parâmetros como composição química [El-Shobokshy and Hussein 1993], propriedades da superfície dos módulos [Hupa et al. 2005], velocidade do vento e até suspensão de partículas de ar [Javed et al. 2017] devem ser levados em consideração conta. A modelagem apresentada em [Coello and Boyle 2019] para estimar a deposição de massa de partículas nos módulos fotovoltaicos é baseada em:

$$
\mathrm{m}=\left(\mathrm{v}_{10-2.5} \mathrm{C}_{10-2.5}+\mathrm{v}_{2.5} \mathrm{C}_{2.5}\right) \mathrm{t} \cos (\theta)
$$

em que, $\mathrm{m}$ é a massa acumulada no intervalo de tempo $\left[\mathrm{g} / \mathrm{m}^{2}\right]$, $\mathrm{t}$ é o intervalo de tempo em segundo, v é a velocidade de deposição do particulado $[\mathrm{g} / \mathrm{m}], \mathrm{C}$ é a concentração do particulado no ambiente $\left[\mathrm{g} / \mathrm{m}^{3}\right], \theta$ é o ângulo de inclinação do módulo fotovoltaico, $10-2.5$ é o índice de particulados correspondente à 10 até $2,5 \mu \mathrm{m}$ e 2.5 é o índice de particulado menor que $2,5 \mu \mathrm{m}$.

A Equação 1 foi ajustada de acordo com as partículas encontradas na usina solar FV utilizada neste trabalho, de forma que os dados correspondentes às partículas acima de 2,5 $\mu \mathrm{m}$ foram descartados (no período sob teste e avaliações, o sensor utilizado não detectou partículas acima desta faixa). Outros ajustes foram necessários ao modelo original, como altura do sensor e inclinação do módulo fotovoltaico.

Durante o período de Outubro de 2019 até Abril de 2020, utilizando a estação de sujidade, realizou-se a coleta de dados 7 ambientais e dos particulados, tanto em quantidade de concentração quanto em massa com tamanho menores que $2.5 \mu \mathrm{m}$. Apesar do período em funcionamento corresponder cerca de 180 dias, por problemas no funcionamento e falhas na padronização das amostras temporais, apenas 105 dias foram aproveitados para análise. A partir dos dados coletados, aplica-se a Equação1 para observar o comportamento do acúmulo de sujidade nos módulos fotovoltaicos. Destaca-se que o efeito da chuva, não foi considerado nas equações de acúmulo de particulados uma vez que tal efeito envolveria novos estudos sobre o impacto da precipitação da chuva na limpeza dos módulos. Os potenciais impactos da chuva sobre os módulos FV serão observados automaticamente pelo sistema de estimativa de geração energética uma vez que a produção real deve ser maior que a produção estimada pelo modelo. Tais situações também podem determinar início de novos períodos cujos dados serão usados para treinamento do modelo.

\section{Modelagem e Desenvolvimento do Preditor Estatístico}

O desenvolvimento do preditor envolve, inicialmente, a determinação de um conjunto de dados que será utilizado para treinamento. Esse conjunto deve corresponder a um período em que os módulos fotovoltaicos estão limpos, possibilitando assim uma produção energética com sua capacidade máxima. Destaca-se que o modelo de preditor tem sua saída indicada em Watts $(\mathrm{W})$ correspondendo à potência em corrente contínua (potência DC). Essa potência é comparada, para fins de avaliação do modelo, com a saída dos módulos fotovoltaicos e entrada do equipamento inversor presente na usina solar fotovoltaica. A decisão sobre a utilização da métrica da potência DC, ao invés da potência AC e energia elétrica produzida pela usina, ocorre visando eliminar os efeitos de erros e perdas do equipamento inversor.

\footnotetext{
${ }^{7}$ Disponível em https://www.kaggle.com/lscadfacomufms/solar2-photovoltaic-solar-plant-data
} 
Pelas observações sobre a geração energética após a limpeza manual dos módulos $\mathrm{FV}$, foi definido um período de 21 dias $(\mathrm{D}=21)$ para conjunto de treinamento. Para cada um dos $\mathrm{D}$ dias, desde a primeira medição de potência gerada no início do dia, a cada $\mathrm{r}=10$ minutos uma nova medição foi realizada até que a planta fotovoltaica não tenha mais capacidade de geração (no final do o dia). Para o d-ésimo dia, obtém-se $n_{d}$ medidas, para $\mathrm{d}=1, \ldots, \mathrm{D}$.

Seja $\mathrm{W}_{\mathrm{id}}$ a soma acumulada das potências geradas até $\mathrm{T}_{\mathrm{id}}$-ésimo instante de tempo, para $\mathrm{i}=1, \ldots, \mathrm{n}_{\mathrm{d}}$ e $\mathrm{d}=1, \ldots, \mathrm{D}$. O mesmo método de aquisição de potência gerada foi adotado para a coleta de dados ambientais como temperatura e irradiância. Sejam $\mathrm{Z}_{\mathrm{id}}$ e $\mathrm{I}_{\mathrm{id}}$, a temperatura e irradiância também no tempo $\mathrm{T}_{\mathrm{id}}$. A massa acumulada $\mathrm{M}_{\mathrm{id}}$ é obtida aplicando a Equação 1 .

Como o preditor estatístico tem como pressuposto básico que não há correlação entre as variáveis explicativas, faz-se necessário calcular a correlação de Pearson para cada par de variáveis. A irradiância e a temperatura têm uma forte relação linear ascendente $(r=0,7103)$. Por outro lado, a irradiância tem correlação fraca com a massa acumulada $(\mathrm{r}=0,2708)$. A temperatura e a massa acumulada tem uma correlação moderada $(r=0,4469)$. Devido à forte correlação linear entre as variáveis irradiância e temperatura, optou-se por desconsiderar a variável temperatura para o ajuste do modelo.

O modelo NLME proposto possui a seguinte relação hierárquica:

$$
\begin{aligned}
\mathrm{Y}_{\mathrm{id}} & =\left(\alpha_{0}+\mathrm{A}_{\mathrm{id}}\right)-\log \left(1+\exp \left\{\left(\beta_{0}+\mathrm{B}_{\mathrm{id}}\right)-\gamma \mathrm{t}_{\mathrm{id}}\right\}\right)+\varepsilon_{\mathrm{id}} \\
\mathrm{A}_{\mathrm{id}} & =\alpha_{1} \mathrm{I}_{\mathrm{id}}+\alpha_{2} \mathrm{M}_{\mathrm{id}}+\mathrm{b}_{\mathrm{id}} \\
\mathrm{B}_{\mathrm{id}} & =\beta_{1} \mathrm{I}_{\mathrm{id}}+\beta_{2} \mathrm{M}_{\mathrm{id}}+\mathrm{c}_{\mathrm{id}}
\end{aligned}
$$

em que, $\left(\mathrm{b}_{\mathrm{id}}, \mathrm{c}_{\mathrm{id}}\right)$ é vetor de efeitos aleatórios assumido como sendo gerado de uma distribuição normal bivariada com variâncias $\sigma_{\mathrm{b}}^{2}$ e $\sigma_{\mathrm{c}}^{2}$ e covariância $\sigma_{\mathrm{bc}}$.

Definido um modelo de efeitos mistos, um dos principais pontos a serem abordados é identificar quais efeitos são importantes. Assim, além do modelo dado na Equação (2), ajustamos outros nove modelos; sendo um modelo sem os efeitos aleatórios, modelos com efeito aleatório em apenas um dos parâmetros, com efeito aleatório em dois parâmetros e com efeito aleatório nos três parâmetros. Além disso, um modelo de regressão linear foi também definido, uma vez que é um modelo padrão usado para estimativas de energia gerada. A estimativa dos parâmetros dos modelos foi realizada com o auxílio da função nlme ${ }^{8}$. Os modelos foram comparados utilizando como critério o erro quadrático médio e os critérios de seleção de modelos AIC 9 e BIC 4 . De acordo com esses critérios, o modelo mais adequado aos dados é o que apresentar menor valor.

O melhor modelo indicado pelos três critérios considerados, não indicou a variável massa como uma variável significativa para explicar a variável resposta (potência gerada). De acordo com o nível de significância adotado para a avaliação, $\omega=\{0.01,0.05\}$, a variável massa (parâmetros $\alpha_{2}$ e $\beta_{2}$ ) foi a que apresentou os menores valores de estimativa conforme a Tabela 1,

Nesse caso, entende-se que a justificativa para não-significância da variável massa

\footnotetext{
${ }^{8}$ Disponível como pacote na linguagem $\mathrm{R}$.

${ }^{9}$ Informação de Akaike [Akaike 1974].

${ }^{10}$ Critério de Informação Bayesiano [Schwarz 1978].
} 
Tabela 1. Estimativas para os parâmetros do modelo da equação 2.

\begin{tabular}{c|c|c|c|c|c}
\hline Parâm. & Estimativas & Erro pad. & Graus de lib. & $\mathrm{t}$-valor & $\mathrm{p}$-valor \\
\hline$\alpha_{0}$ & 3,5968 & 0,0622 & 896 & 57,8062 & $<1 \mathrm{e}-04$ \\
\hline$\alpha_{1}$ & 0,8600 & 0,0057 & 896 & 150,1517 & $<1 \mathrm{e}-04$ \\
\hline$\alpha_{2}$ & $-0,0053$ & 0,0039 & 896 & $-1,3658$ & 0,1723 \\
\hline$\beta_{0}$ & 1,7840 & 0,0827 & 896 & 21,5656 & $<1 \mathrm{e}-04$ \\
\hline$\beta_{1}$ & $-0,2845$ & 0,0081 & 896 & $-35,2973$ & $<1 \mathrm{e}-04$ \\
\hline$\beta_{2}$ & $-0,0160$ & 0,0095 & 896 & $-1,6890$ & 0,0916 \\
\hline$\gamma$ & 0,0429 & 0,0021 & 896 & 20,6064 & $<1 \mathrm{e}-04$ \\
\hline
\end{tabular}

deve-se as características dos dados coletados e repassados para treino e teste do modelo. Com conjuntos de dados maiores e que representam períodos mais longos de tempo é esperado que a massa seja uma variável significativa para explicação da potência gerada. Assim, o modelo selecionado ficou em função da irradiância e do tempo. O modelo ajustado sem a massa apresentou erro quadrático médio de 0,0044 e a sua expressão matemática é dada por:

$$
\hat{\mathrm{Y}}_{\mathrm{id}}=2,7561+0,9398 \mathrm{I}_{\mathrm{id}}-\log \left(1+\exp \left\{-0,6719+0,1134 \mathrm{I}_{\mathrm{id}}-0,2063 \mathrm{t}_{\mathrm{id}}\right\}\right)
$$

para $\mathrm{i}=1, \ldots, \mathrm{n}_{\mathrm{d}}$ e $\mathrm{d}=1, \ldots, \mathrm{D}$.

A partir do modelo ajustado, utilizou-se a diferença percentual (dada pela expressão a seguir) entre a potência observada e a potência esperada (estimada) para analisar as perdas de geração devido à sujidade:

$$
P_{d}=\left(\frac{O_{d}-E_{d}}{E_{d}}\right) \times 100
$$

onde $\mathrm{O}_{\mathrm{d}}=\exp \left\{\mathrm{Y}_{\mathrm{n}_{\mathrm{d}} \mathrm{d}}\right\}$ e $\mathrm{E}_{\mathrm{d}}=\exp \left\{\hat{\mathrm{Y}}_{\mathrm{n}_{\mathrm{d}} \mathrm{d}}\right\}$, para d $=1, \ldots$, D. Se $\mathrm{P}_{\mathrm{d}}<0$, então, a energia gerada no d-ésimo dia foi menor do que o esperado, o que significa que há um impacto de sujidade na geração de energia no d-ésimo dia.

\section{Resultados e Discussões}

Os experimentos e resultados obtidos neste trabalho são baseados na aplicação do preditor sobre o conjunto de dados coletados pela estação de sujidade. A Figura 4 apresenta os valores de saída do preditor em comparação com a potência real (observada) gerada, em um período de testes de 105 dias. Os valores apresentados correspondem à potência DC acumulada (em escala logarítmica) diariamente.

O erro quadrático médio (EQM) geral (ao longo do período de 105 dias) do modelo foi de 0,0044 indicando a proximidade dos valores preditos e observados. Observase alguns períodos em que os valores sugeridos pelo modelo são maiores que os efetivamente gerados. Tais períodos são indicadores da presença de sujidade sobre os módulos, uma vez que essa sujidade faz com que a produção real seja menor do que a estimada, gerando as perdas de geração. De outra forma, estimativas do modelo menores que os valores reais (período entre os dias 5 e 10) sugerem que os módulos estão limpos, correspondendo a períodos após limpeza manual ou natural (via efeito de chuvas) dos módulos.

A Figura 5 apresenta os valores da diferença percentual entre os valores observados e os valores preditos pelo modelo ajustado. Note que uma percentagem positiva neste caso, representa uma maior produção energética pelo sistema fotovoltaico em relação ao 


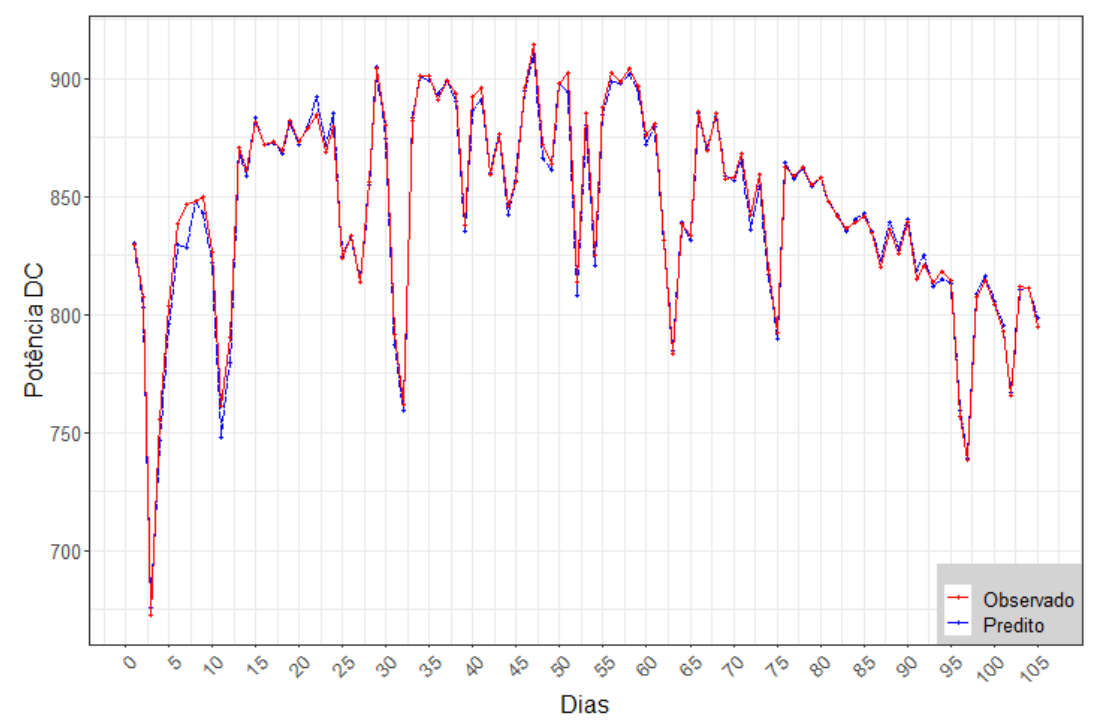

Figura 4. Potências preditas e observadas entre 24/out/2019 e 30/abr/2020.

valor estimado pelo preditor e, para este conjunto de dados, a diferença máxima entre o valor observado e predito foi de $0,9 \%$. Por outro lado, valores negativos na figura representam uma perda na produção energética, onde o valor estimado foi superior ao valor real produzido, sendo essa perda limitada à $1,1 \%$. Observa-se que entre os dias 25 e 80 as diferenças são mínimas, devido ao período de chuvas frequentes sobre a UFV possibilitando assim a limpeza natural dos módulos fotovoltaicos. A partir do dia 80, a diferença percentual média vai se tornando cada vez mais negativa, demonstrando a possível ocorrência de acumulo de sujidade impactando a produção sobre os módulos fotovoltaicos. Diferenças percentuais positivas esporádicas também aparecem no gráfico, e podem ser justificadas por condições atípicas dos fatores ambientais como mudanças na temperatura, dias nublados e mudanças bruscas no clima.

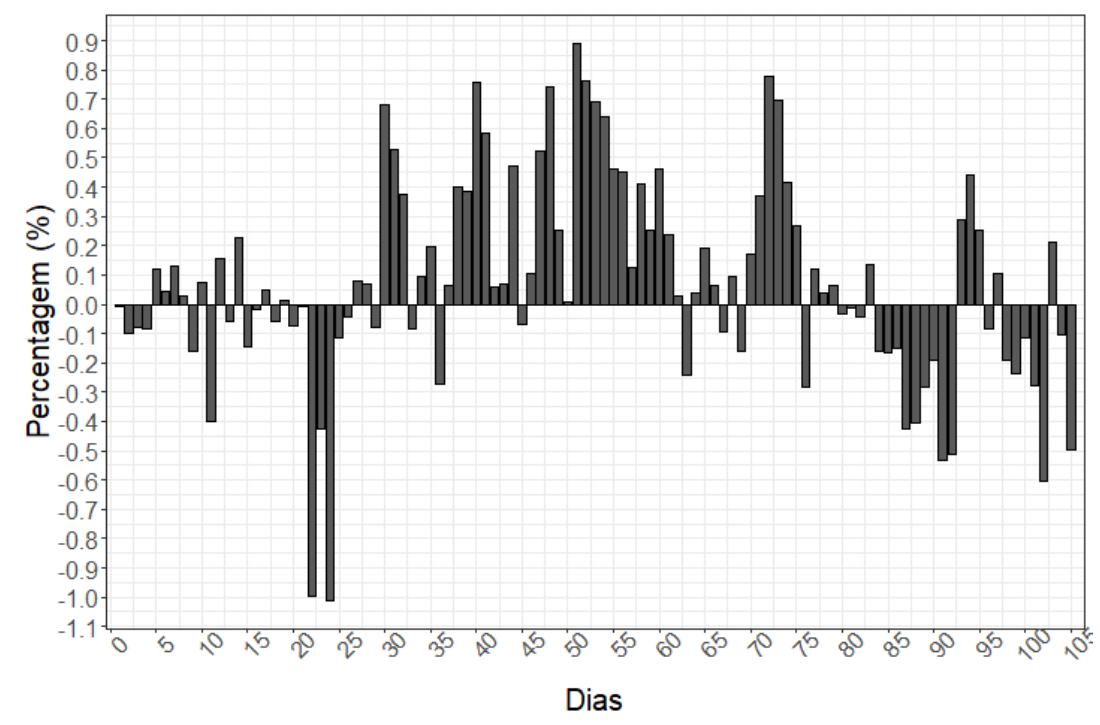

Figura 5. Diferenças percentuais diárias.

\section{Considerações Finais}

Este trabalho apresentou o desenvolvimento de uma solução de hardware e software que possibilita acompanhar a geração de energia e avaliar o impacto de perdas devido à suji- 
dade em uma usina solar fotovoltaica. Esse sistema atende uma demanda existente para a indústria de geração de energia solar fotovoltaica uma vez que oferece automatização e acurácia de predição para embasar a tomada de decisão de manutenção (limpeza) sobre módulos solares fotovoltaicos.

O sistema de hardware/software desenvolvido foi preponderante para a construção de um conjunto de dados ambientais e de geração de energia a partir da Usina Solar Fotovoltaica localizada na UFMS em Campo Grande-MS. Com tais dados, foi possível treinar, testar, validar e avaliar o preditor estatístico desenvolvido. Análises estatísticas sobre o preditor demonstram a viabilidade (coeficiente de correlação $\rho=0,9961$ ) e erros mínimos (erro quadrático médio de 0,0044). De acordo com o preditor, as perdas diárias máximas, no período avaliado, foram de $1,1 \%$. A partir da diferença entre a produção estimada e a produção observada real pode-se mensurar as perdas de geração que, devido à utilização das variáveis ambientais pelo preditor, devem ser atribuídas à presença de particulados (sujidade) sobre os módulos solares fotovoltaicos. O sistema de predição, inclusive, tem sido alvo de estudos para trabalhos futuros seja na avaliação em períodos mais longos assim como em direção à integração desse sistema como fonte de dados para um sistema de viabilidade econômica para tomada de decisão sobre a limpeza dos módulos fotovoltaicos.

\section{Agradecimentos}

Os autores agradecem as companhias distribuidoras CEM, CEC, CEP e o PD-ANEEL pelo suporte financeiro a este trabalho (PD-06961-0007/2017). Também agradecem as agências de fomento: CAPES, CNPq e FUNDECT pelo suporte financeiro ao desenvolvimento de pesquisas no LSCAD/FACOM/UFMS.

\section{Referências}

Akaike, H. (1974). A new look at the statistical model identification. IEEE Transactions on Automatic Control, 19(6):716-723.

Catelani, M., Ciani, L., Cristaldi, L., Faifer, M., Lazzaroni, M., and Rossi, M. (2012). Characterization of photovoltaic panels: The effects of dust. In IEEE International Energy Conference and Exhibition, pages 45-50.

Coello, M. and Boyle, L. (2019). Simple model for predicting time series soiling of photovoltaic panels. IEEE Journal of Photovoltaics, 9(5):1382-1387.

El-Shobokshy, M. and Hussein, F. (1993). Effect of dust with different physical properties on the performance of photovoltaic cells. Solar energy, 6(51):505-511.

Gostein, M., Caron, J. R., and Littmann, B. (2014). Measuring soiling losses at utilityscale pv power plants. In IEEE Photovoltaic Specialist Conference (PVSC), pages 0885-0890.

Hammad, B., Al-Abed, M., Al-Ghandoor, A., Al-Sardeah, A., and Al-Bashir, A. (2018). Modeling and analysis of dust and temperature effects on photovoltaic systems' performance and optimal cleaning frequency: Jordan case study. Renewable and Sustainable Energy Reviews, 82:2218-2234. 
Hupa, L., Bergman, R., Fröberg, L., Vane-Tempest, S., Hupa, M., Kronberg, T., PesonenLeinonen, E., and Sjöberg, A.-M. (2005). Chemical resistance and cleanability of glazed surfaces. Surface science, 584(1):113-118.

Javed, W., Guo, B., and Figgis, B. (2017). Modeling of photovoltaic soiling loss as a function of environmental variables. Solar Energy, 157:397-407.

Mani, M. and Pillai, R. (2010). Impact of dust on solar photovoltaic (pv) performance: Research status, challenges and recommendations. Renewable and Sustainable Energy Reviews, 14:3124-3131.

Mehta, S., Azad, A. P., Chemmengath, S. A., Raykar, V., and Kalyanaraman, S. (2018). DeepSolarEye: Power Loss Prediction and Weakly Supervised Soiling Localization via Fully Convolutional Networks for Solar Panels. Proceedings of the 2018 IEEE Winter Conference on Applications of Computer Vision (WACV), pages 333-342.

Pavan, A. M., Mellit, A., De Pieri, D., and Kalogirou, S. A. (2013). A comparison between bnn and regression polynomial methods for the evaluation of the effect of soiling in large scale photovoltaic plants. Applied energy, 108:392-401.

Ribeiro, K., Santos, R., Saraiva, E., and Rajagopal, R. (2021). A statistical methodology to estimate soiling losses on photovoltaic solar plants. Journal of Solar Energy Engineering, 143(6):064501.

Schwarz, G. (1978). Estimating the dimension of a model. Ann. Statist., 6(2):461-464.

Zorrilla-Casanova, J., Philiougine, M., Carretero, J., Bernaola, P., Carpena, P., MoraLópez, L., and Sidrach-de Cardona, M. (2011). Analysis of dust losses in photovoltaic modules. In World Renewable Energy Congress, pages 8-13. 